\title{
Anthropologie des enjeux de la violence chez lagunaires de Côte d'Ivoire
}

\author{
Mel Meledje Raymond \\ Université de Bouaké - Département d'Anthropologie et de Sociologie \\ O8 B.P. 1033 ABIDJAN 08 \\ Email:m_meledje07@yahoo.fr
}

\section{Résumé}

Le projet d'une Côte d'Ivoire nouvelle suscite particulièrement dans les communautés lagunaires une dynamique de transformation des ordres sociaux (institutions, idéologie des classes d'âge, matrilinéarité) face aux nouveaux enjeux qui mobilisent les populations (démocratie, développement, bien-être des populations, respect des institutions, création de nouvelles richesses, etc.). L'analyse socio-anthropologique de la micro-violence dans ces communautés à travers ces ordres sociaux et leur transformation, la justification des indices d'expression de la violence et les enjeux de celle-ci dans la transformation sociale, révèle que la violence en «s'inscrivant" dans ce processus comme un désordre apparent (Gbudzu-gbudzu), mieux comme un moyen de passage de l'ordre ancien à l'ordre nouveau, est au surplus le thermostat dans la bonne marche de la transformation sociale pour un mieux être.

Mots-clés: Micro-violence, matrilinéarité, classes d'âge, développement, conflit, désordre, transformation,

\section{Introduction}

Dans la littérature des sciences sociales relatives à la Côte d'Ivoire, depuis la crise sociopolitique qui a débuté en 1999, l'on constate un intérêt croissant pour la macro violence (Akindès 1990; Akindès 2003; Akindès 2004; Konaté 2004; Bouquet 2005; Langer 2005; Collett 2006; Marshall-Fratani 2006). Mais, pour comprendre les dynamiques de la macro-violence, l'on ne peut minorer l'importance des micro-violences, c'est-à-dire des expressions de violence socialement et/ou géographiquement circonscrites. Dans le contexte ivoirien l'analyse des micro-violence s'est surtout polarisée sur les enjeux du conflit foncier dans la partie Ouest (Chauveau 2000; Chauveau, Pape et al. 2001; Chauveau 2003; Ibo 2006; Sissoko and Goh 2006; Vanga and Affou 2006) avec une forte implication des jeunes (Chauveau 2003; Ibo 2006) et dans une moindre mesure 
sur le Sud (Colin 1990) et le Nord ( Bassett et al. 1993; 1995; Dugué, Akindès et al.2004; Strozeski 2006) de la Côte d'Ivoire. Or l'analyse socio-anthropologique de la micro-violence dans une Côte d'Ivoire en mutation pourrait aussi s'enrichir de la dynamique de la transformation des ordres sociaux dans des communautés tout aussi traversées par des contradictions sociales. L'objectif du présent article est de comprendre les enjeux de la micro-violence chez les lagunaires de Côte d'Ivoire, des sociétés dont l'organisation et le sens de la hiérarchie sociales semblent être confrontés aux pressions du changement social dont les formes spécifiques d'expression que nous présenterons apparaissent comme étant des indices.

La thèse soutenue dans cet article est la suivante : la violence ouvre le chantier des changements sociaux dans les communautés lagunaires au travers des organisations et des projets de développement local, de la démocratie, du bienêtre des populations, du respect des institutions à une Côte d'Ivoire nouvelle, mais des changements risqués.

Ainsi, dans une première partie, nous allons montrer les ordres sociaux dans les communautés lagunaires et leur transformation. Dans une deuxième partie, nous tenterons de justifier les indices d'expressions de la violence dans ces communautés. Enfin, dans une troisième, nous tenterons d'analyser et de comprendre les enjeux de la violence dans la transformation sociale.

\section{I- Des ordres sociaux et leur transformation}

Les lagunaires dont il est question ici sont ceux des communautés ethniques autochtones principalement Ahizi, Alladian, Ebrié ou Kyaman et Odjoukrou. Ils se sont installés le long du bassin occidental de la lagune ébrié au sud de la Côte d'Ivoire.

Leurs mouvements migratoires sur la bande littorale maritime et lagunaire ont donné lieu à une longue histoire commune de brassage de populations et d'activités de pêche, de commerce et d'agriculture. Il faut souligner que deux souches étaient aux origines du brassage des populations : la souche Akan (venue de l'Est) et la souche krou (venue de l'ouest).

La souche Akan est composée de : Alladian, Ahizi (d'Allaba, Tabot, Atoutou, Nigui-Assoko, Abraniemmiembo, Téfrédji), Odjoukrou (d'Aklodz), Ebrié ou Kyaman (P. Loyer, 1702 ; P. Roussier, 1936 ; Atger, 1962 ; M. Augé, 1968 ; H. Diabaté, 1988 ; R. Allou Kouamé, 2002 ; H. Memel Fotê, 1980 ; J. N. Loukou, 1984 ; J.P. Colleyn, 1986 ; B.G. Diringbin, 1989. La souche Krou est aussi composée de : Ahizi (d'Abra, Nidz et Tchagba), Abidji-Obrou, Odjoukrou (Oborou, Dibmein) et des Kyaman-Abia (H. Diabaté, 1989 ; R. Allou Kouamé, 
2002 ; H. Memel Fotê, 1980). A ces populations il faut ajouter celles venues plus tard de l'intérieur de l'actuelle Côte d'Ivoire et de l'étranger pour faire fortune dans les mêmes activités que les autochtones. Ces populations ont adopté des institutions et des systèmes de parenté presque semblables, par exemple les cas du système des classes d'âge, de la chefferie villageoise et de la matrilinéarité (système de parenté).

\section{I-1- Des ordres sociaux}

\section{I-1-1-Au niveau des institutions}

\section{I-1-1-1- Les classes d'âges}

I-1-1-1-1- Approche conceptuelle

Panoff M. et Perrin M. (1973) définissent la classe d'âge en ces termes "Ensembles d'individus ayant approximativement le même âge, de l'un ou l'autre sexe, et qui sont organisés de manière cohérente en groupe socialement reconnu soumis aux mêmes rites de passage, ayant le même statut au sein de la société globale et exerçant les mêmes activités". Il faut retenir ici deux caractéristiques majeures : le statut de groupe social officiel et l'égalité, fondée sur l'identité de formation, de statut et de fonctions, qui règle les rapports entre membres. Mais en fait, ce sont les rites de passage qui, en définitive, consacrent officiellement les groupes de jeunes recrues et en font des classes d'âge reconnus (Memel-Fotê H., 1980).

Ces rites constituent l'initiation qui est selon Claude Rivière "à la fois épreuve physique, stage de formation à la vie adulte, mode d'accès aux vérités secrètes, permis d'entrer dans la vie sexuelle, intégration à la communauté des vivants et des morts" (1969). Certes, si l'initiation collective exprime un principe d'égalité comme le fait remarquer J.Hurault à propos du système bamiléké : "Aucun principe de biérarchie ne présidait à la formation, et il s'engageait pas non plus, à mesure que les jeunes gens grandissent, la raison d'être de cette institution étant de créer entre eux des liens de solidarité durable, fondée sur une rigoureuse égalité. Non seulement les fils de chefs étaient admis sur le même pied d'égalité que les fils de serviteurs, mais on évitait de créer à l'intérieur de chaque société des fonctions (président, etc.) qui auraient été une source de discorde" (1969), dans bien des cas cette égalité se trouve tempérée par la hiérarchie à l'intérieur de la classe.

En effet, il y a d'abord une hiérarchie d'âge : les aînés et les cadets ; ensuite une hiérarchie de fonctions qui accompagne ce premier type de hiérarchie. Il y a également une hiérarchie entre classes d'âge qui s'exprime au niveau des comportements et du langage en termes de respect qui régit les relations entre aîné et cadet, père et fils. 
Enfin, dans l'organisation des sociétés, les classes d'âge accomplissent des fonctions importantes au bénéfice des membres qu'au bénéfice de la société globale.

Par exemple, au bénéfice des membres, les classes d'âge assurent un rôle de groupes de secours mutuel ; ils y trouvent aide, assistance, bref, solidarité matérielle et morale tout au long de leur existence. Au bénéfice de la société globale, les classes d'âge remplissent une première fonction importante qui est d'ordre économique.

Elles se constituent en main d'œuvre dans la production des biens de subsistance individuels et familiaux, soit "des biens de fêtes" collectives (J. Capron, 1969), soit des biens religieux (confection de vêtements liturgiques). Le rôle politique majeur est celui de la défense de la patrie pour lequel une formation militaire des classes d'âge est exigée.

\section{I-1-1-1-2- Terminologie, nombre et réalité des classes d'âge chez les lagunaires}

\section{I-1-1-1-2-1- Terminologie et nombre}

Les Ahizi-Abra et les Odjoukrou dénomment la classe d'âge Oworn et ont sept classes : obodjlou, sete, ndzroma, abrma, mbedje, mborma, nigbesi. Les Alladian dénomment également la classe d'âge Esouba et ont cinq classes : agrou, atioukou, abobo, aïri, asro. Les Kyaman nomment la classe d'âge Asepasa et en ont quatre: dugbo, niando, breswe, tchagba.

Enfin, pour les Ahizi-Atoutou la classe d'âge est Adro comme chez les AhiziNidz elle est Tchagba. Mais les deux groupes ont chacun douze classes : mbro$a k a$, somo-aka, tchouake, dzipake, bogake, trotchouake, ladzake, martake, leteake, amake, sokpake, atchrake.

\section{I-1-1-1-2-2- Recrutement et formation}

Dans toutes les communautés lagunaires (Ahizi, Alladian, Ebrié et Odjoukrou) l'accès aux classes d'âge et l'égalité de statut de membre se justifient par les conditions sociales et culturelles. L'appartenance à un patrilignage est une condition sine qua non. Entre les classes d'âge, une durée fixe variable d'une société à l'autre constitue l'unité de mesure séparant deux classes d'âge. Par exemple, de huit ans chez les Ahizi-Abra et Odjoukrou ; de trois à cinq ans chez les Alladian; de quinze ans chez les Ebrié. La promotion des jeunes recrues s'effectue réellement dans l'initiation : low en modjoukrou ; esuba-krua en alladian ; gnakpa en ahizi-atoutou. Bref, le but de cette initiation est de pourvoir 
la société en hommes cultivés, en citoyens responsables et en patriotes guerriers. Cette promotion s'opère donc par une formation technique : "l'apprentissage de la guerre" (Memel-Fotê H, 1980:405). Il faut souligner qu'à cet apprentissage se trouvent étroitement associées la formation morale, l'assimilation des vertus nécessaires de solidarité, de discipline et de fidélité auxquelles on confère un caractère sacré.

Marc Augé, à propos de la formation physique, rapporte que chez les Alladian les exercices de combats, destinés à hisser le chef de file (efreno) au sein des classes chargées de la défense armée, se pratiquent au village, au main nue, opposant soit la moitié ouest et la moitié est d'un même esouba, soit deux camps d'esouba apparentés où s'allient esouba-fils, esouba-pères, esouba-grands-pères (1969).

Chez les Odjoukrou, à propos de l'histoire et la pratique de la guerre pendant la formation, Memel-Fôtê H. rapporte également que "la formation bistorique porte spécialement sur l'origine du peuple et les guerres entre tribus. Son but est quadruple: cultiver le patriotisme des jeunes, susciter des héros sur des modèles anciens, assurer la cohésion du groupe et justifier les luttes futures. Chose significative : l'enseignement s'achève par un exercice pratique intitulé "guerre d'initiation". Les initiés ce jour-là, portent leur panoplie, ayant en guise de fusils, des bras de bananiers.

Les récits les soulèvent d'une colère apparente; ils veulent gagner le champ de bataille, les adultes le calment, en vain. Ils enfoncent la cohue et prennent le chemin de la savane, ceux de Usr vers Lokp ou vice versa, ceux de Gbadjn vers Kosr ou vice versa. $A$ Lokp leurs cadets les accompagnent, déguisés en femmes.

Bientôt une pétarade fuse dans l'air ; un guerrier, le plus faible de la promotion, joue au blessé; les "femmes" se lamentent, l'armée regagne le village. $A$ l'entrée des cours, les guerriers subissent un bain rituel et la peinture du caolin blanc" (1980: 324).

I-1-1-1-2-3- Rôle des classes d'âge et signification de l'initiation

Si des conditions sociales et culturelles justifient l'accès aux classes d'âge et l'égalité de statut de membre, le rôle initial à elles destinées est d'ordre militaire : organiser les hommes de vingt à cinquante ans pour la défense de la sécurité et de l'indépendance de la patrie sous la direction des chefs de guerre.

Ensuite vient un rôle économique : travaux pour le village, pour les tuteurs des classes d'âge et entraide entre camarades de promotion ; puis un rôle politique : police du village, pour les aînés sociaux, selon les cas, conseil de gouvernement, fourniture d'armes et de munitions en cas de guerre. Enfin, un rôle culturel distraire la population villageoise par leurs musiciens et chorégraphes.

Bref, à travers les rôles des classes d'âge se dessine en filigrane une signification 
éloquente de l'initiation (qui ouvre aux classes): l'initiation par ses différents rites opère le passage d'une vie à la mort et de la mort à une nouvelle et pleine vie ${ }^{1}$.

Mais en interprétant davantage leur initiation en termes de génération les lagunaires insistent et retiennent le second moment dialectique de la théorie classique à savoir : de la mort à une nouvelle et pleine vie. En effet, les patriclans et les classes monitrices ou "tutrices" accouchent des initiés, au sens culturel; les initiés recréent eux-mêmes leur nouvel être, dans et par les épreuves physiques, intellectuelles et morales qu'ils maitrisent.

Bref, les communautés lagunaires dans leur ensemble se régénèrent périodiquement par l'initiation. C'est pourquoi, l'initiation en classes d'âge est "une fête où les groupes rivalisent de richesses, manifestant leurs capacités économiques et le réseau de leurs relations sociales. Fête des jeunes, fête des clans, fête de la société des vivants et des morts, fête de la nature et de la vie, une réception des rameaux l'ouvre et un partage des rameaux la clôt”, dit Memel-Fotê H (1980 : 326).

Bref, une fois initiés les jeunes gens deviennent ipso facto des citoyens des sociétés qui les portent et assument les fonctions plus haut citées.

Mais le pouvoir suprême (le gouvernement du village), $E b-e b$, chez les Odjoukrou par exemple, est assumé de manière cyclique par les classes d'âge, tous les cinq, huit ou douze ans selon les sociétés ou communautés ethniques.

La prise donc de pouvoir par les seniors leur confèrent ce qu'il est convenu d'appeler "chefferie collective des classes d'âge". Cette chefferie laissera plus tard place à la "chefferie villageoise" initiée par le colonisateur. A ces types de chefferies les lagunaires vouent respect et autorité, honneur et gloire.

\section{I-1-1-2- La chefferie villageois $e^{2}$}

Après avoir soumis tous les résistants à l'action colonisatrice, l'Etat colonial français décida vers 1910 de l'application de l'Administration indirecte à l'ensemble de la Côte d'Ivoire conquise et particulièrement à la région des lagunes dont les formations politiques étaient jusque-là lignagères à classes d'âge.

C'est ainsi qu'il créa des chefferies villageoises en rapport avec les chefferies cantonales (créés également par le colonisateur) et le gouverneur du cercle. Dans un premier temps l'application de cette institution nouvelle ne fut pas heureuse : confusion de rôles et de prérogatives entre la chefferie "collective des classes d'âge" et celles de la nouvelle chefferie villageoise.

1 C'est une théorie classique bien résumée par Claude Lévi-Strauss dans La pensée sauvage (1962 :350) et illustrée par Robert Jaulin dans La mort sara (1967).

2 Voir à cet effet Memel-Fotê H (1980), Augé M (1969), Mel Meledje R (1994). 
Avec le temps, les villageois ont fini par accepter de force ou de gré la nouvelle chefferie. Mais dans la réalité villageoise, le nouveau chef gouverne devant les anciens et ils se doivent mutuellement respect et à leur suite toutes les classes d'âge.

Pour l'Administration coloniale, le chef du village était une "courroie de transmission" entre le gouverneur de la région et le village.

Au village, le chef s'entourait de notables, de héraut(s) et d'anciens de classes d'âge selon la vision institutionnelle de la communauté.

Ainsi, dans les chefferies villageoises lagunaires le sens de l'organisation et de la hiérarchie était toujours sauvegardé et articulé sur les principes du système politique et social des classes d'âge pour lesquels l'idéologie et les structures de fraternité et d'unité fonctionnent. Rappelons brièvement quelques structures idéologiques : le lignage, la classe d'âge, la palabre politique et culte public des morts ${ }^{3}$.

Le lignage (patrilignage ou matrilignage) : au sein du lignage, l'ancêtre éponyme sert de référence à tous et les relations entre membres constituent le lignage en une communauté d'hommes et de femmes dans laquelle dominent des courants solidaires et fraternels.

La classe d'âge : les camarades ou "frères" d'une même renaissance initiatique sont régis par des liens de solidarité et de fraternité qui les soudent les uns aux autres pour la vie.

La palabre politique : assemblée politique des citoyens mâles réunis pour juger, délibérer et décider afin de maintenir l'unité de la communauté. Le culte public des morts : makpegn-ob (odjoukrou), agbamankô (ahizi- Atoutou-Allaba) est une institution cultuelle politico-religieuse (publique) qui restaure annuellement la communauté villageoise.

Donc en réunissant le même jour les vivants et les morts de la communauté villageoise en patrilignages, autour des patriarches pour un repas cultuel et communiel, les parentalies ou le culte public des morts, manifestent à la fois la cohésion des résidents, l'unité et la fraternité de toute la communauté.

A propos de la sauvegarde et du maintien de l'unité et de la fraternité, les anciens qui sont chargés de l'interprétation et de l'application de l'idéologie se révèlent puissants dans l'exécution des décisions.

C'est pourquoi Marc Augé (1974) parle d’idéologique unique parce que celle-ci n'accepte pas des discours, pratiques et interprétations qui lui soient contradictoires.

L'idéologique (langue et idéologie) demeure donc la référence dernière. Emmanuel Terray quant à lui parle d' “espace social à courbure" (1978),

3 Voir Mel Meledje R (1994) et Memel-Fotê H (1980). 
parce que seuls les anciens occupent toute la scène et les autres ne parviennent jamais à se constituer en force autonome, malgré les remous de la démocratie de l'arbre à palabres (Mel Meledje R, 1994 ; Sylla Laciné, 2001).

Bref, il s'agit donc d'une domination sans partage parce que le système en place est assez puissant pour prévenir la formation de concurrents éventuels. Mais l'écran protecteur dont bénéficiait l'idéologique dans "l'espace social à courbure" du temps des anciens n'est plus, il sera remis en cause et même rejeté au temps des jeunes générations et de la modernité. Nous le verrons plus loin avec les transformations.

\section{I-1-2- Au niveau du système de parenté : cas de la matrilinéarité I-1-2-1-Approche conceptuelle et histoire}

Si le matrilignage peut être défini comme un groupe de filiation unilinéaire dont les membres se considèrent comme descendants en ligne utérine d'un ou d'une ancêtre commun(e) et connu (e) et nommé (e), le matrilinéaire se présente comme un système de filiation et d'organisation sociale dans lequel seule l'ascendance maternelle est prise en ligne de compte pour la transmission des privilèges (héritage), d'appartenance à un clan ou à une classe. Alors la matrilinéarité est le "vivre" ou la pratique de ce système de parenté et d'organisation articulé sur la ligne maternelle.

Pour les Akan en général et les Akan lagunaires en particulier, la matrilinéarité est un héritage historique qui s'origine dans la descendance et le mouvement migratoire de la reine Abla Pokou (XVII - XVIII ${ }^{\circ}$ siècle $)^{4}$, nièce et héritière du grand roi Osséi Toutou qui jeta au XVIII ${ }^{\circ}$ siècle les bases de la puissante Confédération aschanti.

Après la mort d'Opokou Waré, jeune frère d'Osséi Toutou, Abla Pokou pour éviter le massacre de ses partisans et sa famille les rassembla et organisa leur fuite vers l'ouest.

Les Akan de Côte d'Ivoire, descendants des Aschanti et héritiers matrilinéaires d'Abla Pokou continuent de vivre les enseignements de la lignée.

Ainsi, chez les Akan lagunaires, la pratique de la matrilinéarité se révèle décisive dans la transmission des privilèges et ses règles incommensurables dans la généalogie matrilinéaire.

Par exemple, la vraie famille de l'enfant est celle de la lignée maternelle. Il peut travailler pour son père, le nourrir, l'enterrer, mais ne l'hérite pas. Il héritera de l'oncle maternel. La femme également peut gouverner et hériter sans

4 Voir particulièrement Jean-Noel Loukou et Françoise Ligier, 1977, La Reine Pokou, Fondatrice du royaume baoulé, Paris, Editions $\mathrm{ABC}$. 
conteste des oncles ou frères de la lignée maternelle quand elle est en rang utile pour le faire. Bref, comme une vérité de Lapalisse, la logique interne de ces ordres sociaux avait convaincu et régi hier les sociétés qui les pratiquaient jusqu'au jour où le système lui-même sera remis en cause par des opposants internes ou externes convaincus à leur tour qu'il faudrait voir et vivre autrement.

\section{1-2- Des transformations}

Les ordres sociaux que nous venons de décrire et d'analyser plus haut sont en fait le résultat d'une longue évolution historique. Mais dans la modernité actuelle qui est aussi le temps des jeunes, ils vont subirent des transformations structurelles importantes et seront même menacés de disparition.

\section{1-2-1-Au niveau des institutions}

Par son organisation, son fonctionnement et sa périodicité, le système des classes d'âge renforçait avec le temps son idéologie sociale et politique de fraternité et d'unité par l'égalité et le dynamisme des membres des classes d'âge. De nouveaux termes comme màl-idj (en odjoukrou) ou Abri (en alladian) expriment cette similitude des rapports entre pairs et ce dynamisme interne des classes et se traduisent par : compagnon, camarade, promotionnaire.

Ensuite, lélargissement de la société politique par l'initiation de nombreux jeunes membres, la collégialisation du pouvoir et la limitation temporelle de son exercice a donné naissance à de nouvelles institutions introduites dans le système politique. Mais le dépérissement de ces nouvelles institutions est déjà entamé par la démocratie, la modernisation, la décentralisation, le développement local en marche dans les nouveaux Etats africains comme dans la Côte d'Ivoire nouvelle. Par exemple, les nouveaux regroupements par affiliation aux partis politiques nationaux et à leurs idéaux, la création de nouvelles Préfectures, Sous-préfectures et Communes comme instruments de développement local, les conflits de générations, soit au niveau des objets de développement, soit au niveau des terres que l'histoire commune a réservée à leur intention, soit au niveau de la hiérarchie sociale face à la pression du changement global...affaiblissent l'unité, l'égalité, le dynamisme et la fraternité des classes d'âge et développent des oppositions souvent farouches entre elles.

La chefferie villageoise sera à son tour frappée de dépérissement avec la création de nouveaux pôles administratifs dans le pays rural : préfectures, souspréfectures, communes dans les villages. Cela entraine la réduction de l'espace d'autorité et de pouvoir des chefs de village. La crédibilité et la compétence de ces 
derniers sont souvent mises à nu par les jeunes générations contestataires. La crise militaro-politique de la Côte d'Ivoire qui a débuté en 2002 a également donné l'occasion de porter aux nues l'incompétence des chefs et rois traditionnels qui prétendaient résoudre la crise ivoirienne au niveau national avec les mécanismes de gestion des conflits dont ils avaient la "maitrise" dans les arènes villageoises 5 .

Bref, la décentralisation de l'Administration par la communalisation des villages inaugure véritablement la mort de la chefferie villageoise.

\section{1-2-2-Au niveau de la matrilinéarité}

Cet ordre social et ses règles qui ont fait autorité hier dans les sociétés lagunaires, agonisent de nos jours sous deux rapports malgré des résistances: le soulèvement des générations jeunes contre les règles de la matrilinéarité (village d'Ousrou en ses débuts) et la loi des successions de la Côte d'Ivoire nouvelle qui stipule en substance : "les enfants héritent de leurs géniteurs père et mère".

Aujourd'hui, dans la réalité villageoise, on peut encore parler d'alternative législative parce que les populations peuvent choisir le mode de législation qui leur convient selon leurs intérêts. Mais les jeunes générations recourent la plus part du temps à la législation étatique pour se rendre justice et réclamer leur dû. Malheureusement ce processus est toujours émaillé de violence entre les opposants.

Bref, les ordres sociaux d'hier se transforment coûte que coûte aujourd'hui face aux pressions d'un environnement lui-même changeant. Aussi ces changements interviennent dans la violence et l'opposition des parties. Alors quelle justification les lagunaires donnent-ils aux indices d'expression de cette violence?

\section{2- Justification des indices d'expression de violence : constats et opportunités}

\section{2-1-Constats de distanciation à la violence dans les communautés lagunaires}

La longue histoire de cohabitation des lagunaires révèle une relative homogénéité manifestée dans les données géographico-écologiques, de l'anthropologie sociale, de l'histoire (Mel Meledje R.) ${ }^{6}$.

\footnotetext{
5 Mel Meledje R., Les rois et chefs traditionnels face à la crise ivoirienne : les mécanismes traditionnels de gestion des conflits sont-ils appropriés? A paraitre.

6 Mel Meledje R., idem.
} 
Celle-ci a donné lieu à des similitudes langagières dont témoignent les productions des expressions de la distance à la violence.

\section{2-I-1- Chez les Abizi}

Les Ahizi nomment de plusieurs manières la violence. Ainsi : $\left.1^{\circ}\right)$ Nôtchu ata : il a l'esprit guerrier ou il aime la guerre; $\left.2^{\circ}\right)$ Nôtchu kucru kucru : il aime la brutalité ou il est brutal; $\left.3^{\circ}\right)$ Napa egbi : il fait tout avec force ou il est dominateur; $4^{\circ}$ )

Gnana ké gbudzu-gbudzu : il est brutal ou il est violent; Gnana ogbo wo mrinmrin: il parle mal ou est violent verbalement.

De la sorte, la brutalité ou la force aveugle exprime la distanciation à la violence. Pêcheurs, les Ahizi sont arrivés aujourd'hui à l'utilisation de cette force comme nécessaire pour faire respecter les règles des activités de pêche sur la lagune et de cohabitation aux contrevenants récidivistes dans les localités.

\section{2-I-2- Chez les Odjoukrou}

Les Odjoukrou disent : $1^{\circ}$ ) Ongn gbudz-gbudz: tu es brutal, violent; $2^{\circ}$ ) Ongn ar: tu possèdes l'esprit guerrier ou tu es dominateur; $3^{\circ}$ ) Bicok ob-fein letch-em ou cokro-cokro-em : tu fais tout avec force ou avec brutalité : tu es belliqueux; $4^{\circ}$ ) Ongn odad: tu aimes la palabre ou la violence verbale.

Certes, plus agriculteurs que pêcheurs, les Odjoukrou étaient jaloux des leurs propriétés comme de leurs héritages. Les matrilignages ne partagent pas, par exemple, leur terre avec les patrilignages. Mais aujourd'hui, les terres des matrilignages odjoukrou sont aussi revendiquées par les descendants des patrilignages comme conforme aux nouvelles dispositions du code civil ivoirien ("L'enfant hérite de ses géniteurs, père et mère"). La charge de violence libérée dans l'altercation est l'un des principaux indices de distanciation sociale de transformation : les anciennes dispositions coutumières deviennent caduques même dans les arènes villageoises régies jusque-là par les règles de la matrilinéarité. Au surplus, une nouvelle race d'Odjoukrou voit le jour.

\section{2-I-3- Chez les Kyaman ou Ebrié}

Les Kyaman désignent par Adi ou Adusan: la violence; Ata : la guerre; Agbobi: la palabre. Par exemple : cet homme est violent, Sèlokon duasanho; cette femme est violente, Biélokon duansanho; pourquoi aimes-tu faire la guerre?, Ebikin è sran- 
manbué nin ? ; Pourquoi, aimes-tu te battre? Ebikin è adibi nin ?; Pourquoi es-tu si violent? Ebikin ètè adusanhi ? En effet, l'utilisation de la violence comme moyen de survie justifie le changement opéré par les Ebrié de notre champ d'étude: devenus de moins en moins pêcheurs, les Ebrié se sont tournés résolument vers la culture du manioc (fabrication de l'atiéké), du palmier à huile et de l'hévéaculture.

Mais la rareté des terres cultivables suscite une course effrénée vers celles disponibles avec quelquefois une violence indescriptible dans l'occupation. Pour ceux qui vaquent encore aux activités de pêche, les plans d'eau lagunaires sont désormais disputés souvent avec violence par des étrangers-pêcheurs et des autochtones. Indice de distanciation sociale de transformation, la violence inaugure les temps de lutte et de réinvention des Ebrié.

\section{2-I-4- Chez les Alladian}

Les Alladian nomment la violence Fru-fru ou Gbudzu-gbudzu. Par exemple : Yesso kanban gbudzu-gbudzu, ce qui veut dire que Yesso fait de la violence ou est violent ; Yesso kanhan frufru, Yesso est devenu brutal, ne respecte plus rien. Certes, si la formation des citoyens Alladian en classes d'âge avait le mérite du respect de l'autorité, de tout homme et du sentiment de responsabilité de toute chose publique et privée, l'esprit de domination, d'influence et de brutalité (frufru ou gbudzu-gbudzu) qui se développe davantage aujourd'hui par la jeunesse alladian peut aussi trouver meilleure explication dans les manifestations de 1990 réclamant l'avènement effectif du multipartisme et de la démocratie au niveau national. La jeunesse ivoirienne dans son ensemble s'en est impliquée et a même payé le lourd tribut (plusieurs morts). Aujourd'hui encore elle continue la lutte même dans les localités les plus éloignées sous l'égide de "l'Association des patriotes". Dans les villages alladian cette jeunesse est désormais au devant de toutes les manifestations et récriminations sociales et politiques, mais aussi des prises de position qui entrainent la violence. Certes, indice de distanciation sociale pour un ordre nouveau, la violence manifestée par la jeunesse alladian ouvre la voie à l'Alladian nouveau.

Bref, de cette production sociale de l'expression de la violence chez les lagunaires et des commentaires qui ont suivi, nous pouvons faire les remarques suivantes : - des similitudes langagières constatées attestent de la longue cohabitation des populations et au surplus de leur relative homogénéité. D'ailleurs, il est également constaté que le terme Gbudzu-gbudzu commun à toutes les communautés lagunaires est le résultat d'une évolution après cokro-cokro, $k r u$ kru, fru-fru, fian-fian. Il exprime d'une certaine façon l'intensité plus grande 
de la violence ; - l'accroissement de la violence et son intensité plus grande ces dernières années dans ces localités sont fonction de l'émergence de nouveaux défis comme les connaissances nouvelles, l'ouverture aux autres horizons, à la démocratie, au développement local, à l'emploi et au refus de l'ancien système dominé par la matrilinéarité ; - le développement de l'hévéaculture dans la région des lagunes entraine des conflits violents dans l'occupation des terres et les nouveaux regroupements qu'induit la décentralisation de l'Administration territoriale. Certes, ces facteurs dont nous revisiterons quelques uns dans les "Opportunités" influencent à la fois le langage dans la production sociale d'expression théorique de distanciation de la violence et dans une large mesure, les comportements. Alors qu'en est-il donc des Opportunités de la violence sur le terrain?

\section{2-2-Les Opportunités de distanciation à la violence dans les communautés lagunaires}

La vie dans les communautés villageoises et les relations entre des communautés lagunaires révèlent: pour les communautés qui vivent déjà éloignées les unes des autres dans le même village, les fréquentations comme les services réciproques régressent ; pour les communautés ethniques, les problèmes de frontières comme ceux du foncier ressurgissent ; pour celles qui habitent la bande littorale, la réfection des infrastructures communes et les appuis économiques des populations deviennent objets de palabres et de violence.

\section{2-2-I- Chez les Ahizi}

\section{Au niveau local: non respect des lois de cohabitation}

Le village d'Allaba abrite depuis plusieurs années une population étrangère de pêcheurs (Béninois et Togolais) devenue plus nombreuse que la population autochtone. Les lois internes qui les régissaient hier sont devenues caduques aux yeux de la génération montante autochtone et étrangère née dans le village. Ainsi, le non-respect des lois de la communauté sont réprouvées sévèrement par "les soldats" de la chefferie ou des anciens. A cet effet, la violence verbale ou physique exercée ces dernières années (2005-2008) pour mettre au pas les contrevenants est inhabituelle. Désormais l'impunité n'est plus acceptée au village. Et on entend les autochtones dire aux étrangers "Si vous ne voulez pas accepter nos nouvelles lois, il vaut mieux entrer chez vous!". Cette atmosphère met en mal l'harmonie du village et les services réciproques. 
Le village d'Atoutou-A avait accepté, il y a plusieurs années, à la demande des étrangers de leur accorder à titre gracieux la presqu'île du village pour habitation et activités de pêche. Cette demande a été acceptée. Depuis lors, les relations entre les deux communautés étaient plutôt harmonieuses. Mais voilà qu'aujourd'hui, le village d'Atoutou-A érigé en commune depuis 2007, se voit contraint de réviser le contrat d'occupation de la presqu'île établi à titre gracieux. Désormais les étrangers de la presqu'île doivent payer à la municipalité des redevances ou l'impôt d'habitation.

Et c'est le refus d'exécution de bon nombre d'entre eux qui provoqua la colère de la population autochtone : des menaces d'expulsion et de violence verbale et physique ont été enregistrées de part et d'autre. Depuis lors, les relations entre les communautés sont empruntes de méfiance.

Des étrangers ont même quitté le village et ceux qui y sont restés vivent dans la peur de se voir chasser un jour pour un impôt impayé ou pour une agression (verbale ou physique) portée à un autochtone.

\section{Au niveau des relations entre villages autochtones : conflits fonciers}

Les villages de Nidz-Assoko et Nianmiembo se disputent un espace terrien pour lequel ils se réclament tous deux propriétaires bien avant l'indépendance du pays. Mais très occupés par les activités de pêche, les Ahizi de Nidz-Assoko ont mis en veilleuse cette revendication. Aujourd'hui, avec l'essor de l'hévéaculture dans la région, la course à la terre pour des villages voisins distants l'un de l'autre d'environ 600 mètres, devient source de conflits violents. A chaque occasion, les deux villages libèrent une charge de violence instrumentale qui fait des morts. Ainsi, malgré la réconciliation des deux villages, la méfiance s'en est installée jusqu'aujourd'hui.

Les villages d'Allaba (Ahizi) et de Ngatti (Odjoukrou) séparés l'un de l'autre d'environ 300 à 500 mètres par endroit, se disputent depuis plusieurs années le chef de terre de l'espace des deux villages, du moins, le premier arrivé en ces lieux. A chaque altercation les deux villages libèrent une charge de violence qui fait des morts de part et d'autre. La voie principale de sortie terrestre du village d'Allaba est celle passant par Ngatti, alors que ce dernier monte des barrages pendant les périodes de crise, empêchant les habitants d'Allaba d'avoir facilement accès à la ville de Dabou. Et c'est bien une des raisons du ralliement d'Allaba à la nouvelle commune de Tabot. Mais les conflits entre les deux n'ont jamais eu de cesse.

\section{Au niveau du développement : revendication d'instruments économiques}

Le bac à péage de Jacqueville dit aussi bac de N'djêm est devenu un instrument 
économique convoité par la nouvelle commune de Sassako (village alladian) et celle homologue de Tabot (village ahizi). La revendication de cet instrument par l'une et l'autre commune a suscité de vives controverses et de violences qui ont amené l'armée ivoirienne à séjourner pendant un mois dans la région (en 2008).

Le ré-profilage de la route d'Allaba-Dabou en passant par Ngatti (en 2009) qui exigeait de rabaisser la colline de Ngatti côté Allaba a été l'objet de vives tensions entre les deux villages (Allaba et Ngatti). Une fois encore, la bagarre violente qui s'en est suivie est terminée par la mort d'hommes. La police nationale, la brigade de gendarmerie et le conseil général de la région de Dabou ont volé au secours des villages. Mais malgré les solutions communes arrêtées, les deux villages se regardent toujours en chiens de faïence.

\section{2-2-2-Chez les Odjoukrou}

\section{Au niveau local : problèmes politique, économique et socio-culturel}

Pendant de la crise ivoirienne, des jeunes étrangers Maliens, Burkinabé, Guinéens et autochtones odjoukrou, armes à la main, attaquent et volent les habitants du village d'Ousrou B, de nuit comme de jour, sur les chemins des champs comme dans les maisons. Excédés, les habitants du village s'organisent avec toutes sortes d'armes et attendent les "prédateurs". Au signal donné par le héraut du village, un échange de coup de feu fait des morts principalement dans le camp des "prédateurs" parmi lesquels se trouvent des fils du village identifiés. Depuis cette attaque des villageois, Ousrou B s'est débarrassé des voleurs armés, mais reste vigilent.

Le village de Toupa (village odjoukrou) abrite un nombre important d'étrangers (Burkinabé, Maliens, Guinéens, Sierra-Leonniens), ouvriers agricoles dans les plantations de palmiers à huile, d'hévéa et salariés à l'usine de transformation du latex de l'hévéa du village et à l'huilerie de palme de Cosrou (village odjoukrou) et commerçants. Pendant les événements de la crise (en 2002), des étrangers Burkinabé auraient pris partie pour Alassane Dramane Ouattara, président du parti du Rassemblement des Républicains (R. D. R). De nuit, ils montent des barrages et agressent les passants (automobilistes, passagers...). Mais voilà qu'un soir, les villageois, la police nationale et la gendarmerie y ont donné l'assaut pour sécuriser les lieux. La charge de violence libérée a fait plusieurs morts parmi les étrangers insurgés. Depuis cette nuit, on n'a plus entendu parler d'agresseurs Burkinabé dans la région, mais la méfiance entre étrangers Burkinabé et autochtones Odjoukrou s'en est installée. 
Au niveau des relations entre villages autochtones : problèmes économique, de frontière

Le village de Lopou (village odjoukrou) fait frontière avec ceux de Yassap-A et $\mathbf{B}$ (villages odjoukrou). Les problèmes frontiers mettent en conflit d'une année à l'autre les trois villages. Ces dernières années (en 2005), un match de football entre jeunes des dits villages a dégénéré en bataille rangée. La création du village de Yassap B et l'extension des plantations d'hévéa du nouveau village ont fait l'objet d'empiètement constaté par le village de Lopou, mais sans violence à l'époque. Ainsi, à la suite de la bataille rangée entre jeunes footballeurs et la poursuite des jeunes de Lopou par les habitants des deux villages Yassap jusqu'audelà des limites de leurs frontières ont alerté les habitants de Lopou qui ont chargé à leur tour les deux villages Yassap. La guerre déclenchée a été fatale des deux côtés : des morts. Depuis cet événement malheureux, ces villages voisins sont sur le "qui vive" aux frontières respectives et les relations entre Lopou et les villages Yassap ne sont plus au beau fixe.

Les villages odjoukrou voisins d'Okpoyou-Em et d'Oursou A et $\mathbf{B}$ cohabitent très difficilement. Aux origines, Ousrou $\mathbf{B}$ et Opkoyou-Em font partie de l'unique village d'Ousrou (A). Et plus tard pour des raisons démographiques et d'entente, Oursou B et Okpoyou-Em sont créés et installés "pas trop loin et pas très près" d'Oursou A. Mais, bien que partis, les habitants d'OkpoyouEm veulent encore conserver une partie des terres d'Ousrou A. Pire, ils louent cette terre à un industriel Chinois qui y crée une vaste plantation d'hévéa. La guerre déclarée est impitoyable, surtout qu'Okpoyou-Em refuse de reverser une partie des redevances à lui octroyées au village d'Ousrou A. La charge de violence libérée par les deux villages d'Ousrou contre Okpoyou-Em est fatale : des morts par centaines du côté d'Okpoyou-Em. Depuis ces événements (2007), les coopérations comme les relations entre les Ousrou-A et B et Okpoyou-Em sont rompues. Mais des expéditions punitives perpétrées sur les routes et dans plantations ont été enregistrées de part et d'autre ces dernières années.

Au niveau du développement: problème économique et de remise en cause de l'autorité

Le village de Débrimou (village odjoukrou) crée une vaste plantation collective d'hévéa pour financer les projets de développement local. Et la gestion de cette plantation est confiée par le village aux classes d'âge gouvernantes. Cette annéelà, c'est la classe d'âge Mborman au pouvoir suprême qui gère le patrimoine. Et voici qu'un projet de réhabilitation de l'école primaire et de canalisation des eaux de pluie exige un décaissement d'argent. L'assemblée villageoise commande alors aux gestionnaires Mborman un bilan à mi-parcours avant le décaissement. 
Le bilan est catastrophique : un déficit de 250 millions de francs cfa est constaté. Pour plus de clarté et de crédibilité, à nouveau, le village fait appel à un cabinet d'expertise comptable. Le résultat de l'expertise est formel : un déficit de 250 millions de francs cfa. Alors la colère des villageois monte au paroxysme. Les anciens calment les esprits surchauffés ; puis donnent un mois aux gestionnaires pour rembourser la dette. Mais voilà que le délai expire et que le village n'est pas entré dans ses fonds. Alors il décide de punir sévèrement par tous les moyens l'équipe de la direction pour servir de leçon aux classes d'âge futures gestionnaires.

Des actes sont joints à la parole : les premiers décès dans l'équipe provoquentl'émoi et la frayeur dans la population villageoise. Mais entretemps, le décaissement d'argent est fait et les travaux ont été réalisés.

Dans les villages odjoukrou généralement, la réalisation d'un projet de développement communautaire met en compétition financière toutes les classes d'âge. Et c'est à l'occasion de la réhabilitation de l'ancien château d'eau que le village d'Agbaille devient le théâtre d'une vague de violence entre classes d'âge. Les générations montantes souhaitent "la construction d'un nouveau château d'eau plus grand et moderne, puisque la population s'accroît et que le village s'étend de plus en plus". Les anciens trouvent qu'il y a urgence : "Le village a besoin d'eau dans l'immédiat, puisque le château d'eau actuel ne fonctionne plus. Le nouveau projet dont vous parlez est onéreux et ne peut être réalisé dans l'immédiat compte-tenu de nos moyens".

Après la comparaison des devis des deux projets, les générations montantes estiment que "la rébabilitation sera une erreur. Il vaut mieux faire un bon travail une fois pour toutes et se répartir la somme entre classes comme nous avons l'babitude pour la construction d'un nouveau château d'eau'.

Cette proposition des générations montantes ne plait pas aux anciens qui jugent que les jeunes les défient et remettent en cause leur autorité : "Vous remettez toujours en cause nos décisions, méfiez-vous! Et cette fois, cela ne se passera pas comme vous l'entendez!" déclare le doyen d'âge au nom des anciens. Et de discussion en discussion, on en arrive aux bagarres, puis aux rixes avec une charge de violence inhabituelle des générations jeunes. On compte de nombreux blessés parmi les anciens qui ne cessent de maudire les jeunes générations et réclament leur exclusion du village. Depuis cet événement, la tension reste vive entre les anciens et les générations montantes.

Au niveau de la démocratie : problème d'exclusion et recentrement des groupes

Les élections législatives, municipales et présidentielles se déroulent partout en Afrique et particulièrement dans les villes et villages dans une ambiance 
emprunte de violence parce que la culture de démocratie à l'occidental n'est pas encore ancrée dans les mœurs. Tel a été le cas aux élections municipales et législatives démocratiques à Dabou de 1980 et $1985^{7}$.

Aux élections municipales de 1980, au deuxième tour du scrutin, restent en liste Nomelagne Michel, Odjoukrou, originaire de Dibrm, donc de la Commune de Dabou, mais résidant habituellement à Abidjan ; Kol Memel Jean, originaire d'Usr, village de la Sous-préfecture de Dabou, résidant habituellement à Dabou et fondateur du collège Nouvelle Atlantide de la dite ville. Mais Kol Memel est traité d'utu (étranger) par la majorité des Odjoukrou des villages de la Commune, électeurs de Nomelagne.

Aux résultats, Kol Memel est élu. Cette élection lui a coûté une jambe brisée et une hanche fracturée, à cause des hostilités dont il fut l'objet. Les dessous du terme 'étranger' attribué à Kol Memel font remonter la compréhension plus loin : en dehors du découpage administratif de Commune et de Sous-préfecture, l'animosité entre la Confédération de Dibrm et celle de Bobor dont fait partie le village d'origine de Kol Memel va également resurgir.

Aux élections municipales de 1985, au deuxième tour de scrutin, restent en liste : Nomelagne Michel, originaire de Dibrm de la Commune de Dabou, résidant à Abidjan et soutenu par la grande majorité des habitants de Dibrm et alliés ; Lohoues Vincent, originaire de Dibrm de la Commune de Dabou, résidant à Abidjan et soutenu par une partie des habitants de Dibrm, mais en majorité par les habitants du village de Kpas de la dite Commune et alliés.

Il faut souligner qu'en soutenant fortement la candidature de Lohoues Vincent (marié également à une fille de ce village) les habitants du village de Kpas mûrissaient le projet de la candidature de Mangou-Eİ Jean Marie, originaire de Kpas aux législatives.

Aux résultats Lohoues Vincent est élu et de nouveau Nomelagne Michel est battu. Aux législatives, au deuxième tour de scrutin, Lohoues Vincent et Mangou-EÏ Jean-Marie se portent candidats contre Nomelagne Michel.

Il faut souligner que les électeurs de Nomelagne Michel traitent Lohoues Vincent de traitre parce qu'il fait alliance avec un fils d'étranger ou un fils d'esclave (père de Mangou-EÏ J. Marie serait un esclave, dit-on). Aux résultats Nomelagne Michel est élu.

\section{2-2-3- Chez les Kyaman ou Ebrie}

Au niveau des relations entre villages autochtones : problème de frontières

7 Cf. Mel Meledje R., 1994 : 225-228. 


\title{
Anthropologie des enjeux de la violence chez lagunaires de Côte d'Ivoire
}

\author{
Mel Meledje Raymond \\ Université de Bouaké - Département d'Anthropologie et de Sociologie \\ O8 B.P. 1033 ABIDJAN 08 \\ Email:m_meledje07@yahoo.fr
}

\section{Résumé}

Le projet d'une Côte d'Ivoire nouvelle suscite particulièrement dans les communautés lagunaires une dynamique de transformation des ordres sociaux (institutions, idéologie des classes d'âge, matrilinéarité) face aux nouveaux enjeux qui mobilisent les populations (démocratie, développement, bien-être des populations, respect des institutions, création de nouvelles richesses, etc.). L'analyse socio-anthropologique de la micro-violence dans ces communautés à travers ces ordres sociaux et leur transformation, la justification des indices d'expression de la violence et les enjeux de celle-ci dans la transformation sociale, révèle que la violence en «s'inscrivant" dans ce processus comme un désordre apparent (Gbudzu-gbudzu), mieux comme un moyen de passage de l'ordre ancien à l'ordre nouveau, est au surplus le thermostat dans la bonne marche de la transformation sociale pour un mieux être.

Mots-clés: Micro-violence, matrilinéarité, classes d'âge, développement, conflit, désordre, transformation,

\section{Introduction}

Dans la littérature des sciences sociales relatives à la Côte d'Ivoire, depuis la crise sociopolitique qui a débuté en 1999, l'on constate un intérêt croissant pour la macro violence (Akindès 1990; Akindès 2003; Akindès 2004; Konaté 2004; Bouquet 2005; Langer 2005; Collett 2006; Marshall-Fratani 2006). Mais, pour comprendre les dynamiques de la macro-violence, l'on ne peut minorer l'importance des micro-violences, c'est-à-dire des expressions de violence socialement et/ou géographiquement circonscrites. Dans le contexte ivoirien l'analyse des micro-violence s'est surtout polarisée sur les enjeux du conflit foncier dans la partie Ouest (Chauveau 2000; Chauveau, Pape et al. 2001; Chauveau 2003; Ibo 2006; Sissoko and Goh 2006; Vanga and Affou 2006) avec une forte implication des jeunes (Chauveau 2003; Ibo 2006) et dans une moindre mesure 
Le village de Songon-Mbratté (village Ebrié) a maille à partir avec celui de Tabot (village Ahizi), quelques années après l'indépendance de la Côte d'Ivoire.

La délimitation des frontières lagunaires a été remise en cause par des jeunes pêcheurs Ebrié de Songon-Mbratté. Ceux de Tabot trouvent que les repères sont bien à leur place et qu'il ne faudrait pas les déplacer. La discussion entre jeunes pêcheurs Ebrié et Ahizi tourne bien vite en palabre, puis en rixe. Dans la foulée, les pêcheurs Ahizi emmènent avec eux un pêcheur Ebrié. Les habitants de Songon-Mbratté l'ayant appris, descendent sur la lagune, la traversent et détruisent le campement de Ndjêm qui était sous le contrôle des Ahizi de Tabot. Devant cette provocation, les villages Ahizi de Koko et d'Allaba s'invitent au côté de Tabot dans la bataille engagée avec Songon-Mbratté. La charge de violence libérée par les Ahizi sur la lagune oblige les Ebrié à se replier chez eux. Plus tard la conciliation entamée par les anciens Ebrié et Ahizi a permis une visibilité plus grande des frontières lagunaires. Mais les relations entre les Ebrié de Songon-Mbratté et les Ahizi de Tabot sont jusqu'aujourd'hui empruntes de méfiance.

\section{Au niveau du développement : problème économique et de leadership}

Bien avant la crise ivoirienne (2002), la construction d'un marché vaste et moderne divise les Ebrié de Songon-Dagbé et de Songon-Agban. Auparavant, le seul marché de Songon-Agban accueillait, le jour du marché (le dimanche), les vendeuses de poisson du littoral lagunaire et maritime, les ouvriers agricoles des plantations industrielles de banane particulièrement des forêts de Songon et les ménages des villages de Songon-Kassemgbré, Songon-Dagbé, SongonTé, Songon-Agban, Songon-Mbratté, Abiaté et même de Dabou. L'espace marchand s'avère aujourd'hui restreint pour tant de monde. Avec la création de la commune de Songon, il a été jugé bon de délocaliser le marché en un lieu plus vaste et plus sécurisé. Et voilà que le nouveau marché construit à Songon-Dagbé n'est pas du goût de bon nombre des populations, a fortiori, celle de Songon-Agban. L'instigateur principal du refus, Songon-Agban est mal vu par son voisin Songon-Dagbé.

La crise entre ces deux villages trouve son point culminent lors de la négociation entamée par la nouvelle municipalité. De la salle de réunion avec des procès d'intention et de la violence verbale et physique aux villages, la crise s'amplifie.

Le village de Songon-Agban fait construire à ses dépens des hangars modernes sur son marché pour abriter les commerçants. La fidélité des commerçants au marché de Songon-Agban accroît l'abandon du marché de Songon-Dagbé et ravive la haine entre ces deux villages.

Dans le même élan de crises qui secouent les villages Ebrié lagunaires de 
notre champ d'étude, la nouvelle mairie des Songon construite à Adiapoto- 1 est même abandonnée par les nombreux désaccords manifestés avec violence entre eux au profit des anciens bureaux de la SAPH (société africaine de plantations d’hévéa) à Anguédédou.

\section{2-2-4 - Chez les Alladian}

Au niveau des relations entre communautés : non respect des lois de cohabitation

Les relations entre la communauté villageoise autochtone de Sassako et celle étrangère du campement béninois situé sur le territoire villageois de Sassako ne sont pas au beau fixe. Les nombreuses palabres entre communautés ont d'ailleurs été à l'origine de leur séparation. Maintenant qu'elles vivent "pas trop près ni pas trop loin" l'une de l'autre, pensions-nous qu'elles s'entendraient mieux; mais bien au contraire, ce sont des palabres et des conflits à la moindre occasion entre les générations : celle des adultes pendant les activités de pêche et celle des écoliers sur le chemin de l'école. Devenue une commune aujourd'hui (2008), le villagecommune de Sassako souhaite l'installation d'une brigade de gendarmerie ou d'une police nationale pour faire respecter les lois de cohabitation et assainir les environs. En attendant, la méfiance est de mise entre ces deux communautés.

Au niveau du développement: revendication non contrôlée et problème de délocalisation

L'installation de la société de râpage de noix de coco, SICOR à Jacqueville bien avant la crise ivoirienne faisait de cette ville à la fois un pôle d'emploi régional et un instrument économique important pour les populations du littoral. Mais comme par enchantement, la crise qui frappait la Côte d'Ivoire toute entière l'a aussi affectée: un violent mouvement ouvrier de revendication de salaire et de conditions de travail (incendie des locaux) s'est soldé par le licenciement d'une partie du personnel, bagarre en chaine entre les ouvriers, fermeture de l'usine de Jacqueville et enfin délocalisation à Grand-Lahou. Le littoral Alladian connait aujourd'hui un crucial problème d'emploi, puisque même la nouvelle usine d'extraction de pétrole installée récemment au large de Jacqueville ne peut servir de sauvetage : elle n'emploie que des ouvriers hautement qualifiés et le plus souvent venus d'ailleurs; les ouvriers autochtones qualifiés ou non sont très peu nombreux.

Bref, au regard des cas rapportés, les opportunités de distanciation à la violence (Gerwalt) dans les communautés lagunaires sont nombreuses. Et elles 
expriment tantôt le non-respect des lois de cohabitation, les conflits fonciers et frontaliers, tantôt la remise en cause de l'autorité des anciens et les problèmes de leadership, tantôt des revendications non contrôlées de salaires et d'instruments économiques, tantôt une démocratie mal comprise.

De la sorte, en s'inscrivant dans des situations historiques des lagunaires, la violence s'ancre dans la mémoire collective et rend sa résolution difficile (morts d'hommes). Par conséquent, ce qu'elle veut enseigner n'est plus entendu par la partie adverse. Alors elle devient destructrice de la communauté, surtout qu'elle ne connait pas le moment alchimique qui la transforme en force qui rétablit l'ordre ${ }^{8}$. Ainsi, ces problèmes évoquées à travers les constats et les opportunités de distanciation à la violence, les lagunaires cherchent à se réinventer $(\mathrm{S}$. Berstein et M. Winock, 2003) dans une Côte d'Ivoire nouvelle d'économie de marché et de droit ; mais une réinvention tout de même risquée (F. Akindès, 2007).

En effet, pendant longtemps, nombre de ces communautés de pêcheurs comme les Ahizi ne se contentaient que d'activités de pêche. L'agriculture n'était pratiquée que secondairement comme culture de subsistance pour la famille.

Mais voilà que l'hévéaculture en se développant dans la région provoque aussi des changements ou renversements de situation importants: de la primauté accordée hier à la pêche, les lagunaires pénètrent aujourd'hui le continent et font de l'agriculture leur principale activité pourvoyeuse de richesse.

Mais la brutalité avec laquelle ils s'accaparent les terres soient-disant restantes ou s'en réclament la propriété accroît l'hostilité entre eux. De même les structures de développement ou d'appui économique que la Côte d'Ivoire nouvelle inaugure dans la région deviennent objets de lutte et de violence.

Mais la lutte (Kamp) et la violence (Gerwalt) ne sont jamais pensées sans ce rapport au droit qui les transfigure (Azoumana Ouattara, 2007). Dans le cas d'espèce, le droit (dans les sociétés traditionnelles) serait ce qui légitime la propriété ou l'acquisition du premier occupant. Alors, les problèmes frontaliers seraient résolus sans violence par les contractants ou les témoins d'alors.

Or ce qui est donné de constater aujourd'hui semble être dans bien des cas une remise à flot des acquis d'hier ; ce qui engendre une course effrénée et une brutalité dans l'occupation parce quégalement les liens historiques ont été déliés au profit d'intérêts égoïstes ou individuels.

\section{3-Les enjeux de la violence dans la transformation sociale}

Dans les cas rapportés, les causes de la violence ne sont pas perceptibles au moment des faits.

8 Cf. Colliot-Thélène C, «Violence et contrainte », in Lignes, n²5, mai, 1995. 
Leur latence et leur enracinement socioculturel créent des îlots de tension dont l'éclosion constitue ce qu'il est convenu d'appeler violence.

Mais quelque soit l'impact de cette violence sur les individus ou sur les groupes, ceux-ci sont à la fois "auteurs et victimes".

Ainsi, la relation de causalité se fait de plus en plus sentir même dans les termes ou les expressions qui expriment la violence - Nôtchu cru-cru, Gnanaké gbudzu-gbudzu; Ongn gbudzu-gbudzu; Adi, Adusan et fait plus penser à une réaction. Cette réaction si brutale soit-elle est le plus souvent l'œuvre des jeunes générations qui se propulsent au-devant de la scène ; ce qui fait d'eux les principaux acteurs des nouveaux enjeux.

De la sorte, si la violence chez les lagunaires a été perçue comme domination, trafic d'influence et d'autorité et soumission, mais son but pour les nouveaux enjeux est plutôt d'amener le récalcitrant ou le résistant à changer de comportement, de mode de vie et à entrer dans l'ordre nouveau que de rechercher uniquement son anéantissement même si dans l'exercice de la violence la mesure n'est pas toujours dictée.

Donc, en produisant de la violence qui manifeste un désordre apparent dans ces communautés, les jeunes générations ouvrent la voie au changement et aux nouveaux enjeux par un nouvel ordre.

Ces enjeux sont pour les lagunaires : démocratie, bien -être des populations, respect des lois et des organisations, de la chose publique et privée, paix et développement. De la sorte, le désordre et l'ordre comme indicateurs du processus d'ouverture (III-1) aux nouveaux enjeux (III-3) par des mutations profondes (III-2) méritent aussi d'être analysés.

\section{3-1- Désordre et Ordre}

"Vous remettez toujours en cause nos décisions! Et cette fois cela ne se passera pas comme vous l'entendez?", déclare le doyen d'âge au nom des anciens du village d'Agbaille ${ }^{10}$ aux jeunes générations.

La tradition est communément admise comme génératrice de continuité ; elle exprime la relation au passé et sa contrainte ; elle impose une conformité résultant d'un code du sens et donc des valeurs qui régissent les conduites individuelles et collectives transmises de génération en génération.

Elle est un héritage qui définit et entretient un ordre en effaçant l'action transformatrice du temps.

9 Voir également Françoise Ngendahayo (1997).

10 Voir plus haut; Agbaille est un village odjoukrou de nouvelle Commune de Toupa. 
Elle ordonne dans tous les sens et pèse sur le cerveau des hommes comme une 'obsession' (G. Balandier, 1988 : 36). Les choses doivent donc se passer comme l'entendent les anciens !

Mais, ces décisions qui ordonnent et marquent le sens inaltérable des choses sont génératrices de désordre dans l'histoire nouvelle des jeunes générations.

Ainsi, ces dernières utilisent la force et la violence pour s'imposer et s'exprimer ou dire aux anciens que les temps et les expériences ont évolués, qu'un nouvel ordre des choses est établi.

Ce qui revient à dire que pour les jeunes générations la tradition en voulant donner forme et sens au présent devient source de désordre.

Alors, croyons-nous que les anciens nont-ils pas une conscience claire de la présence du désordre et du péril d'immobilisme qui empêcherait toute marche en avant ? Ils en ont bien conscience, mais les dispositions du nouvel esprit et les moyens conséquents leurs manquent. C'est pourquoi nous redit Balandier : " $a$ tradition n'est ni ce qu'elle semble être, ni ce qu'elle dit être; les anthropologues le savent désormais" (1988).

- "Si vous ne voulez pas accepter nos nouvelles lois, il vaut mieux entrer chez vous!", intiment les jeunes générations du village d'Allaba ${ }^{11}$ aux membres de la communauté étrangère récalcitrants ou résistants au nouvel ordre.

Né du désordre, l'ordre nouveau doit se "traditionnaliser" pour se maintenir et s'équilibrer. Ainsi, si la force des jeunes générations provient du travail antérieur des anciens comme texte ou comme patrimoine sur lequel elles veulent bâtir l'avenir, la brutalité avec laquelle elles enseignent ce nouvel ordre doit faire plutôt place à la modération afin de comprendre elles -mêmes le difficile passage qu'elles commandent aux étrangers.

En effet, habitués à respecter l'ordre ancien, ces étrangers entrent brusquement dans le chaos ou le point zéro (ut-topos) de renaissance où se conjuguent ordre, désordre et violence pour donner naissance à un nouvel ordre pour de nouveaux enjeux. Bref, comme l'avers et le revers d'une médaille, l'ordre et le désordre sont indissociables dans la dynamique même de la société.

"Dans une société de tradition qui se définit elle-même en termes d'équilibre, de conformité, de stabilité relative, qui se voit comme un monde à l'endroit, le désordre devient une dynamique négative engendrant un monde à l'envers. Il n'est pas pour autant ignoré que l'inversion de l'ordre n'est pas son renversement (...)", dit G.Balandier (1988). 


\section{3-2- Mutations ou nouveaux enjeux dans la transformation sociale}

- La construction d'un nouveau château d'eau plus grand et moderne est nécessaire parce que la population s'accroît et que le village s'étend.

- L'hévéaculture et la culture du palmier en plein essor ont fini par séduire les lagunaires devenus plutôt des agriculteurs que des pêcheurs. Mais l'occupation des terres se fait avec violence.

- Les habitants du village de Songon-Agban refusent le projet de délocalisation de leur marché pour Songon-Dagbé où l'espace et la sécurité sont acceptables et suffisants pour accueillir les villages voisins. Ce refus crée de l'animosité entre voisins (Songon-Dagbé et Songon-Agban).

- La gestion du bac-navette à péage de Jacqueville est revendiquée par les communes de Sassako et de Tabot, parce qu'opérant sur leur espace commun. Les revendications à titre exclusif se font avec violence.

- La paix entre les communautés villageoises.

- Respect et bon usage des institutions ou structures de développement : nouveau découpage administratif (conseil général, préfecture, sous-préfecture, commune) et regroupements conséquents.

- La démocratie se veut aussi un instrument pour résorber de beaucoup les conflits tribaux et régionaux et favoriser la mobilité des populations dans une Côte d'Ivoire nouvelle. Mais voilà quelle crée chez certains une mentalité de rejet, d'exclusion et de recentrement.

Ces enjeux nouveaux (déjà relevées plus haut) s'inscrivent dans les processus de transformation ou de mutation des ordres sociaux de la région lagunaire comme "bouleversement des systèmes" (Roger Bastide, 1969). En effet, les termes de mutation et de transformation (au sens sociologique) s'inscrivent dans un champ sémantique plus élargi incluant en quelques manières : changement, évolution, révolution, développement, modernisation, etc.

Ils servent le plus souvent à exprimer les différences qui apparaissent par rapport à la stricte répétition des formes sociales; les uns soulignant la pertinence d'une identité fondamentale malgré les modifications apparentes, les autres la rupture (G.Balandier, 1971 et 1986) et non la destruction.

C'est pourquoi le mouvement des ouvriers de la SICOR de Jacqueville a été réprimé sévèrement : licenciement et délocalisation. C'est aussi pourquoi, en parlant de mouvement social plutôt que conscience de classes A.Touraine (1992) préfère un acteur collectif dont une orientation majeure est la défense du sujet, la lutte pour les droits et la dignité des travailleurs.

Ce mouvement était réservé aux ouvriers qualifiés, défenseurs du travail et de 
l'autonomie des ouvriers, comme celui de la SICOR (mouvement des syndiqués et qualifiés). De la sorte, pour A. Touraine, "un mouvement social est à la fois un conflit social et un projet culturel. Cela est vrai de celui des dirigeants comme de celui des dirigés. Il vise toujours à la réalisation des valeurs culturelles en même temps qu'à la victoire sur un adversaire social. Une lutte revendicative n'est pas en elle-même un mouvement social ; elle peut être défense corporative, utilisation de la conjoncture sur le marché du travail, pression politique même.

Pour quelle devienne mouvement social, il faut quelle parle au nom de la société industrielle et s'en fasse le défenseur contre ses propres adversaires. Pas de mouvement social dans la société industrielle tant que les ouvriers s'opposent à l'industrialisation, cassent les machines ou résistent à des techniques nouvelles, même quand c'est pour des raisons importantes et légitimes, dès lors que ces techniques menacent leur emploi; pas de mouvement social non plus si l'action syndicale n'est pas positivement dirigée vers le renforcement de l'autonomie ouvrière et ne bat pas, en particulier, la brutale affirmation des patrons tayloristes : on ne paie pas pour penser"( 1992 : 279).

Bref, les enjeux nouveaux (démocratie, bien-être, respect des lois et des institutions, paix et développement) nécessitent des changements. Et la violence consécutive comme moyen contraignant certes, à l'avènement du nouvel ordre, est le thermostat de la bonne marche dans la transformation ou la mutation sociale.

Pour ainsi dire, les nouveaux enjeux mobilisent, orientent et éclairent le processus de transformation sociale pour un mieux être dont la place de la violence est déterminante.

\section{Conclusion}

Au terme de notre analyse, il apparait que la violence dans les communautés lagunaires a pris du regain et une intensité inhabituelle parce que les amarres sont jetées, les liens déliés (la matrilinéarité est récusée et le système des classes d'âge agonie) et les enjeux attrayants. Les indices d'expression de cette violence ont d'abord révélé des similitudes langagières éloquentes du fait d'une longue cohabitation des communautés; ensuite, elle a insisté sur l'intensité de la violence due à l'émergence de nouveaux enjeux (démocratie, développement local, communalisation, respect des institutions et des personnes, bien-être social) et des sources nouvelles de création de richesses (hévéaculture, bac à péage, emploi salarié). Les implémentations rapportées de la distanciation à la violence ont aussi révélé une brutalisation des communautés dans l'occupation des terres dites inoccupées ou celles que l'histoire commune a réservées à leur intention et de la remise en cause des acquis d'hier dont les règles de la matrilinéarité et l'idéologie 
des systèmes des classes d'âge. Mais l'analyse socio-anthropologique de la micro-violence dans les communautés lagunaires a plutôt révélé la violence non seulement comme un moyen de passage de l'ordre ancien au nouvel ordre, mais davantage comme le thermostat de la bonne marche de la transformation sociale pour un mieux être. Au surplus les enjeux attrayants ouvrent le chantier social, économique et politique d'une réinvention risquée des populations lagunaires à une Côte d'Ivoire nouvelle. Ainsi revient-il aux lagunaires de se maintenir dans cette réinvention en se réappropriant réellement les instruments.

\section{Bibliographie}

Akindès (F), 2003, "Côte d'Ivoire: socio-political crises, ivoirité and the course of history", in African Sociological Review 7 (2):11-28

Akindès (F), 2007, "La crise ivoirienne ou la réinvention risquée d'une nation", in Repères "International", Penser la crise ivoirienne, Paris, Editions Menaibuc-France, novembre.

Allou Kouamé (R), 2002, Histoire des peuples de civilisation AKAN, des origines à 1874, Thèse de Doctorat d'Etat, T.II, Abidjan, Université de Cocody.

Augé (M), 1962, Le rivage alladian, organisation et évolution des villages alladian, Paris, Mémoire de l'ORSTOM, n³4.

Augé (M), 1968, "Temps et société : le cas de la société alladian", in Cahiers ORSTOM, Serie Sciences Humaines, vol., n ${ }^{\circ} 3$.

Azoumana Ouattara, 2007, "La crise du monopole de la violence instrumentale en Côte d'Ivoire", in Repères "International", Penser la crise ivoirienne, Paris, Editions Menaibuc-France, novembre.

Balandier (G), 1988, Le désordre, Eloge du mouvement, Paris, Fayard.

Bastide (R), 1969, "Le problèmes des mutations religieuses", in Cahier international de sociologie, vol. XLVI.

Berstein (S) et Winock (M), 2003, L'invention de la démocratie, Paris, Seuil.

Chauveau (JP), 2000, "La question foncière et construction nationale en Côte d'Ivoire", in Politique Africaine (78) : 94-125.

Chauveau (JP), 2003, "Crise forestière, crise de la ruralité et relations entre autochtones et migrants sahéliens en Côte d'Ivoire" in Conflict and Policy in the Mano River region and Côte d'Ivoire : the regional stakes for stability and reconstruction, Paris, OCDE.

Colin (JP), 1990, Les mutations d'une économie de plantation en basse 
Côte d'Ivoire, Paris, ORSTOM.

Collett (M), 2006, "Ivoirian identity constructions: Ethnicity and Nationalism in the prelude of civil war", in Nations and nationalism 12 (4) : 613-629.

Colliot-Thélène (C), 1995, "Violence et contrainte", in Lignes, n²5, mai.

Diabaté (H), 1988, Eglise et société africaine, Paroisse St Pierre de Jacqueville, un siècle d'apostolat, Abidjan, Les nouvelles Editions

Ibo Guéhi (J), 2006, "Retraits des terres par les jeunes autochtones sur les anciens fronts pionniers de Côte d'Ivoire : expression d'une crise de transition intergénérationnelle", Colloque international sur Les frontières de la question foncière- At the frontier of land issues, Montpellier, 2006, Montpellier-IRD.

Loukou (JN), 1984, Histoire de la Côte d'Ivoire, Formation des peuples, Abidjan, CEDA, T.1.

Marshall-Fratani (R), 2006, "The war of "Who si Who" : autochtony, nationalism and citizenship in the Ivorians crisis", African Review.

Mel Meledje (R), Anthropologie des étrangers et des mutations du vivre-ensemble dans les sociétés lagunaires (alladian, ahizi, Kyaman, odjoukrou), à paraître.

Memel Fotê (H), 1980, Le système politique de Lodjoukrou, une société lignagère à classes d'âge de Côte d'Ivoire, Paris, Abidjan, Dakar, Lomé, Présence Africaine et les Nouvelles Editions Africaines.

N'guendahayo (F), 1997, "Jeunesse et culture de paix : crise urbaine, jeunesse et violence", in Conflits actuels et culture de paix, Actes du colloque d'Abidjan, Abidjan, PUCI.

Touraine (A), 1992, Crise de la modernité, Paris, Fayard.

Wieviorka (M), 2004, La violence, Paris, Editions Balland. 